\title{
q-Supercongruences modulo the fourth power of a cyclotomic polynomial via creative microscoping
}

\author{
Victor J. W. Guo \\ School of Mathematics and Statistics, Huaiyin Normal University, Huai'an 223300, Jiangsu, People's \\ Republic of China \\ jwguo@hytc.edu.cn
}

\begin{abstract}
By applying Chinese remainder theorem for coprime polynomials and the "creative microscoping" method recently introduced by the author and Zudilin, we establish parametric generalizations of three $q$-supercongruences modulo the fourth power of a cyclotomic polynomial. The original $q$-supercongruences then follow from these parametric generalizations by taking the limits as the parameter tends to 1 (l'Hôpital's rule is utilized here). In particular, we prove a complete $q$-analogue of the (J.2) supercongruence of Van Hamme and a complete $q$-analogue of a "divergent" Ramanujan-type supercongruence, thus confirming two recent conjectures of the author. We also put forward some related conjectures, including a $q$-supercongruence modulo the fifth power of a cyclotomic polynomial.
\end{abstract}

Keywords: q-congruence; supercongruence; cyclotomic polynomial; Chinese remainder theorem.

AMS Subject Classifications: 33D15, 11A07, 11B65

\section{Introduction}

In his second notebook, Ramanujan mysteriously recorded 17 infinite series representations of $1 / \pi$ (see [1, p. 352]), including for instance

$$
\sum_{k=0}^{\infty}(6 k+1) \frac{\left(\frac{1}{2}\right)_{k}^{3}}{k !^{3} 4^{k}}=\frac{4}{\pi},
$$

which he later published in [19]. Here and throughout the paper, $(a)_{n}=a(a+1) \cdots(a+$ $n-1)$ stands for the Pochhammer symbol. It was noticed by Van Hamme [24] in 1997 that several Ramanujan's and Ramanujan-type formulas possess nice $p$-adic analogues, such as

$$
\begin{aligned}
& \sum_{k=0}^{(p-1) / 2}(4 k+1) \frac{\left(\frac{1}{2}\right)_{k}^{4}}{k !^{4}} \equiv p \quad\left(\bmod p^{3}\right), \\
& \sum_{k=0}^{(p-1) / 2}(6 k+1) \frac{\left(\frac{1}{2}\right)_{k}^{3}}{k !^{3} 4^{k}} \equiv(-1)^{(p-1) / 2} p \quad\left(\bmod p^{4}\right),
\end{aligned}
$$


where $p>3$ is a prime. The supercongruence (1.1) was proved by Van Hamme [24, (C.2)] himself. Later Long [15] proved that both (1.1) and (1.2) are true modulo $p^{4}$. It was not until 2016 that Van Hamme's last supercongruence was confirmed by Osburn and Zudilin [17] using the WZ method [25]. For more Ramanujan-type supercongruences, see Zudilin's famous paper [26].

During the past few years, many congruences and supercongruences have been generalized to the $q$-settings by different authors (see, for example, [3, 14, 16, 21,23]). In particular, using the $q$-WZ method [25] the author and Wang [13] established a $q$-analogue of (1.1): for odd $n$,

$$
\sum_{k=0}^{(n-1) / 2}[4 k+1] \frac{\left(q ; q^{2}\right)_{k}^{4}}{\left(q^{2} ; q^{2}\right)_{k}^{4}} \equiv q^{(1-n) / 2}[n]+\frac{\left(n^{2}-1\right)(1-q)^{2}}{24} q^{(1-n) / 2}[n]^{3} \quad\left(\bmod [n] \Phi_{n}(q)^{3}\right) .
$$

They [13, Conjecture 5.1] also asserted that the above $q$-congruence is also true when the sum on the left-hand side is over $k$ from 0 to $n-1$. Here and in what follows we adopt the standard $q$-hypergeometric notation: $(a ; q)_{n}=(1-a)(1-a q) \cdots\left(1-a q^{n-1}\right)$ is the $q$-shifted factorial; $[n]=[n]_{q}=1+q+\cdots+q^{n-1}$ is the $q$-integer; and $\Phi_{n}(q)$ denotes the $n$-th cyclotomic polynomial in $q$ :

$$
\Phi_{n}(q)=\prod_{\substack{1 \leqslant k \leqslant n \\ \operatorname{gcd}(n, k)=1}}\left(q-\zeta^{k}\right)
$$

where $\zeta$ is an $n$-th primitive root of unity.

Moreover, the author and Zudilin [14] devised a method, called "creative microscoping", to prove many $q$-supercongruences modulo $\Phi_{n}(q)^{3}$ by adding one or more extra parameters and considering asymptotics at roots of unity. Later the author and Schlosser [10] applied the creative microscoping method to deduce many other $q$-supercongruences from transformation formulas for basic However, no $q$-supercongruences modulo $\Phi_{n}(q)^{4}$ are proved by the creative microscoping method up to now.

In this paper, we shall give a creative microscoping proof of (1.3). More precisely, we shall establish the following parametric generalization of (1.3).

Theorem 1.1. Let $n$ be a positive odd integer. Then, modulo $[n] \Phi_{n}(q)\left(1-a q^{n}\right)\left(a-q^{n}\right)$,

$$
\begin{aligned}
& \sum_{k=0}^{(n-1) / d}[4 k+1] \frac{\left(a q ; q^{2}\right)_{k}\left(q / a ; q^{2}\right)_{k}\left(q ; q^{2}\right)_{k}^{2}}{\left(a q^{2} ; q^{2}\right)_{k}\left(q^{2} / a ; q^{2}\right)_{k}\left(q^{2} ; q^{2}\right)_{k}^{2}} \\
& \quad \equiv q^{(1-n) / 2}[n]+q^{(1-n) / 2}[n] \frac{\left(1-a q^{n}\right)\left(a-q^{n}\right)}{(1-a)^{2}}\left(1-\frac{n(1-a) a^{(n-1) / 2}}{1-a^{n}}\right),
\end{aligned}
$$

where $d=1,2$.

By l'Hôpital's rule, we have

$$
\lim _{a \rightarrow 1} \frac{\left(1-a q^{n}\right)\left(a-q^{n}\right)}{(1-a)^{2}} \frac{\left(1-a^{n}-n(1-a) a^{(n-1) / 2}\right)}{\left(1-a^{n}\right)}=\frac{\left(n^{2}-1\right)(1-q)^{2}}{24}[n]^{2} .
$$


Thus, taking the limits of the two sides of (1.4) as $a \rightarrow 1$, we see that (1.3) is true modulo $\Phi_{n}(q)^{4}$. But the proof of [10, Theorem 12.9] (or [14, Theorem 4.2]) already indicates that it is also true modulo $[n]$. Therefore, the $q$-congruence (1.3) and [13, Conjecture 5.1] are consequences of (1.4).

Likewise, there is a $q$-analogue of (1.2) (i.e., the (J.2) supercongruence of Van Hamme 24]) proposed by the author [5, Conjecture 1.1]: for odd $n$,

$$
\begin{aligned}
& \sum_{k=0}^{(n-1) / 2} q^{k^{2}}[6 k+1] \frac{\left(q ; q^{2}\right)_{k}^{2}\left(q^{2} ; q^{4}\right)_{k}}{\left(q^{4} ; q^{4}\right)_{k}^{3}} \\
& \quad \equiv(-q)^{(1-n) / 2}[n]+\frac{\left(n^{2}-1\right)(1-q)^{2}}{24}(-q)^{(1-n) / 2}[n]^{3} \quad\left(\bmod [n] \Phi_{n}(q)^{3}\right) .
\end{aligned}
$$

The author [5] proved that (1.5) is true modulo $[n] \Phi_{n}(q)$, and the author and Zudilin [14] proved that (1.5) is also true modulo $[n] \Phi_{n}(q)^{2}$ by the aforementioned method of creative microscoping. Here we shall completely confirm (1.5) by showing the following parametric generalization.

Theorem 1.2. Let $n$ be a positive odd integer. Then, modulo $[n] \Phi_{n}(q)\left(1-a q^{n}\right)\left(a-q^{n}\right)$,

$$
\begin{aligned}
& \sum_{k=0}^{(n-1) / 2} q^{k^{2}}[6 k+1] \frac{\left(a q ; q^{2}\right)_{k}\left(q / a ; q^{2}\right)_{k}\left(q^{2} ; q^{4}\right)_{k}}{\left(a q^{4} ; q^{4}\right)_{k}\left(q^{4} / a ; q^{4}\right)_{k}\left(q^{4} ; q^{4}\right)_{k}} \\
& \quad \equiv(-q)^{(1-n) / 2}[n]+(-q)^{(1-n) / 2}[n] \frac{\left(1-a q^{n}\right)\left(a-q^{n}\right)}{(1-a)^{2}}\left(1-\frac{n(1-a) a^{(n-1) / 2}}{1-a^{n}}\right) .
\end{aligned}
$$

It is clear that the $q$-congruence (1.5) modulo $\Phi_{n}(q)^{4}$ follows from (1.6) by taking the limit as $a \rightarrow 1$. Since (1.5) modulo $[n]$ has already been given in [5] and [14], the $q$-congruence (1.5) is thus a direct conclusion of (1.6).

Partially motivated by a supercongruence of Sun [20, Conjecture 5.1(ii)], the author [7, Conjecture 7.1] made the following conjecture: for odd $n$,

$$
\begin{aligned}
& \sum_{k=0}^{n-1}[3 k+1] \frac{\left(q ; q^{2}\right)_{k}^{3} q^{-\left(\begin{array}{c}
k+1 \\
2
\end{array}\right)}}{(q ; q)_{k}^{2}\left(q^{2} ; q^{2}\right)_{k}} \\
& \quad \equiv q^{(1-n) / 2}[n]+\frac{\left(n^{2}-1\right)(1-q)^{2}}{24} q^{(1-n) / 2}[n]^{3} \quad\left(\bmod [n] \Phi_{n}(q)^{3}\right) .
\end{aligned}
$$

The $q$-congruence (1.7) modulo $[n] \Phi_{n}(q)^{2}$ was proved by the author [7] himself, and was reproved by the author and Zudilin [14] by establishing its parametric generalization. Here we shall prove that (1.7) is true by the method of creative microscoping again. Namely, we shall establish the following $q$-congruence. 
Theorem 1.3. Let $n$ be a positive odd integer. Then, modulo $[n] \Phi_{n}(q)\left(1-a q^{n}\right)\left(a-q^{n}\right)$,

$$
\begin{aligned}
& \sum_{k=0}^{n-1}[3 k+1] \frac{\left(a q ; q^{2}\right)_{k}\left(q / a ; q^{2}\right)_{k}\left(q ; q^{2}\right)_{k} q^{-\left(\begin{array}{c}
k+1 \\
2
\end{array}\right)}}{(a q ; q)_{k}(q / a ; q)_{k}\left(q^{2} ; q^{2}\right)_{k}} \\
& \quad \equiv q^{(1-n) / 2}[n]+q^{(1-n) / 2}[n] \frac{\left(1-a q^{n}\right)\left(a-q^{n}\right)}{(1-a)^{2}}\left(1-\frac{n(1-a) a^{(n-1) / 2}}{1-a^{n}}\right) .
\end{aligned}
$$

As before, we can deduce that (1.7) is true modulo $\Phi_{n}(q)^{4}$ (and is therefore also true modulo $\left.[n] \Phi_{n}(q)^{3}\right)$ from (1.8) by taking $a \rightarrow 1$.

The rest of the paper is organized as follows. We shall prove Theorems 1.1 1.3 in Sections 2-4, respectively. More concretely, we shall establish first the corresponding $q$-congruences modulo $[n]\left(1-a q^{n}\right)\left(a-q^{n}\right)\left(b-q^{n}\right)$, where Chinese remainder theorem is utilized. Then Theorems 1.1 1.3 immediately follow from these $q$-congruences with parameters $a$ and $b$ by letting $b \rightarrow 1$. Finally, we propose some related conjectures for further study in Section 5 .

\section{Proof of Theorem 1.1}

We first require the following easily proved lemma.

Lemma 2.1. Let $n$ be a positive odd integer. Then

$$
\begin{aligned}
\left(a q^{2}, q^{2}\right)_{(n-1) / 2}\left(q^{2} / a, q^{2}\right)_{(n-1) / 2} & \equiv(-1)^{(n-1) / 2} \frac{\left(1-a^{n}\right) q^{-(n-1)^{2} / 4}}{(1-a) a^{(n-1) / 2}} \quad\left(\bmod \Phi_{n}(q)\right), \\
\left(a q, q^{2}\right)_{(n-1) / 2}\left(q / a, q^{2}\right)_{(n-1) / 2} & \equiv(-1)^{(n-1) / 2} \frac{\left(1-a^{n}\right) q^{\left(1-n^{2}\right) / 4}}{(1-a) a^{(n-1) / 2}} \quad\left(\bmod \Phi_{n}(q)\right) .
\end{aligned}
$$

Proof. It is easy to see that

$$
\begin{aligned}
\left(q^{2} / a, q^{2}\right)_{(n-1) / 2} & =\left(1-q^{2} / a\right)\left(1-q^{4} / a\right) \cdots\left(1-q^{n-1} / a\right) \\
& \equiv\left(1-q^{2-n} / a\right)\left(1-q^{4-n} / a\right) \cdots\left(1-q^{-1} / a\right) \\
& =(-1)^{(n-1) / 2}\left(a q, q^{2}\right)_{(n-1) / 2} \frac{q^{-(n-1)^{2} / 4}}{a^{(n-1) / 2}} \quad\left(\bmod \Phi_{n}(q)\right) .
\end{aligned}
$$

Therefore, the left-hand side of (2.1) is congruent to

$$
(-1)^{(n-1) / 2}(a q ; q)_{n-1} \frac{q^{-(n-1)^{2} / 4}}{a^{(n-1) / 2}} .
$$

The proof of (2.1) then follows from the fact

$$
(a q ; q)_{n-1}=\sum_{k=0}^{n-1}(-1)^{k} q^{\left(\begin{array}{c}
k+1 \\
2
\end{array}\right)}\left[\begin{array}{c}
n-1 \\
k
\end{array}\right] a^{k} \equiv \sum_{k=0}^{n-1} a^{k} \quad\left(\bmod \Phi_{n}(q)\right),
$$

where we have used the $q$-binomial theorem in the above equality.

Similarly, we can prove (2.2). 
Recall that the author and Zudilin [14, Theorem 4.2] proved the following $q$-congruence:

Lemma 2.2. Let $n$ be a positive odd integer. Then, modulo $[n]\left(1-a q^{n}\right)\left(a-q^{n}\right)$,

$$
\sum_{k=0}^{(n-1) / d}[4 k+1] \frac{\left(a q ; q^{2}\right)_{k}\left(q / a ; q^{2}\right)_{k}\left(q / b ; q^{2}\right)_{k}\left(q ; q^{2}\right)_{k}}{\left(a q^{2} ; q^{2}\right)_{k}\left(q^{2} / a ; q^{2}\right)_{k}\left(b q^{2} ; q^{2}\right)_{k}\left(q^{2} ; q^{2}\right)_{k}} b^{k} \equiv \frac{(b / q)^{(n-1) / 2}\left(q^{2} / b ; q^{2}\right)_{(n-1) / 2}}{\left(b q^{2} ; q^{2}\right)_{(n-1) / 2}}[n],
$$

where $d=1,2$.

We find that the left-hand side of (2.4) also has a simple expression modulo $b-q^{n}$.

Lemma 2.3. Let $n$ be a positive odd integer. Then, modulo $b-q^{n}$,

$$
\sum_{k=0}^{(n-1) / d}[4 k+1] \frac{\left(a q ; q^{2}\right)_{k}\left(q / a ; q^{2}\right)_{k}\left(q / b ; q^{2}\right)_{k}\left(q ; q^{2}\right)_{k}}{\left(a q^{2} ; q^{2}\right)_{k}\left(q^{2} / a ; q^{2}\right)_{k}\left(b q^{2} ; q^{2}\right)_{k}\left(q^{2} ; q^{2}\right)_{k}} b^{k} \equiv \frac{\left(q ; q^{2}\right)_{(n-1) / 2}^{2}[n]}{\left(a q^{2} ; q^{2}\right)_{(n-1) / 2}\left(q^{2} / a ; q^{2}\right)_{(n-1) / 2}}
$$

where $d=1,2$.

Proof. Note that Jackson's ${ }_{6} \phi_{5}$ summation formula can be written as

$$
\sum_{k=0}^{N} \frac{\left(1-a q^{2 k}\right)(a ; q)_{k}(b ; q)_{k}(c ; q)_{k}\left(q^{-N} ; q\right)_{k}}{(1-a)(q ; q)_{k}(a q / b ; q)_{k}(a q / c ; q)_{k}\left(a q^{N+1} ; q\right)_{k}}\left(\frac{a q^{N+1}}{b c}\right)^{k}=\frac{(a q ; q)_{N}(a q / b c ; q)_{N}}{(a q / b ; q)_{N}(a q / c ; q)_{N}}
$$

(see [2, Appendix (II.21)]). Letting $q \mapsto q^{2}$ and taking $a=q, b=a q c=q / a$ and $N=(n-1) / 2$ in the above formula, we obtain

$$
\sum_{k=0}^{(n-1) / 2}[4 k+1] \frac{\left(a q ; q^{2}\right)_{k}\left(q / a ; q^{2}\right)_{k}\left(q^{1-n} ; q^{2}\right)_{k}\left(q ; q^{2}\right)_{k}}{\left(a q^{2} ; q^{2}\right)_{k}\left(q^{2} / a ; q^{2}\right)_{k}\left(q^{n+2} ; q^{2}\right)_{k}\left(q^{2} ; q^{2}\right)_{k}} q^{n k}=\frac{\left(q^{3} ; q^{2}\right)_{(n-1) / 2}\left(q ; q^{2}\right)_{(n-1) / 2}}{\left(a q^{2} ; q^{2}\right)_{(n-1) / 2}\left(q^{2} / a ; q^{2}\right)_{(n-1) / 2}}
$$

Namely, when $b=q^{n}$ both sides of (2.5) are equal, thus establishing this $q$-congruence.

Proof of Theorem 1.1. It is clear that the polynomials $[n]\left(1-a q^{n}\right)\left(a-q^{n}\right)$ and $b-q^{n}$ are relatively prime. By Chinese reminder theorem for relatively prime polynomials, we can determine the remainder of the left-hand side of (2.4) modulo $[n]\left(1-a q^{n}\right)\left(a-q^{n}\right)\left(b-q^{n}\right)$ from (2.4) and (2.5). To accomplish this, we need the following $q$-congruences:

$$
\begin{aligned}
\frac{\left(b-q^{n}\right)\left(a b-1-a^{2}+a q^{n}\right)}{(a-b)(1-a b)} & \equiv 1 \quad\left(\bmod \left(1-a q^{n}\right)\left(a-q^{n}\right)\right), \\
\frac{\left(1-a q^{n}\right)\left(a-q^{n}\right)}{(a-b)(1-a b)} & \equiv 1 \quad\left(\bmod b-q^{n}\right) .
\end{aligned}
$$


Therefore, combining (2.4) and (2.5) we obtain

$$
\begin{aligned}
& \sum_{k=0}^{(n-1) / d}[4 k+1] \frac{\left(a q ; q^{2}\right)_{k}\left(q / a ; q^{2}\right)_{k}\left(q / b ; q^{2}\right)_{k}\left(q ; q^{2}\right)_{k}}{\left(a q^{2} ; q^{2}\right)_{k}\left(q^{2} / a ; q^{2}\right)_{k}\left(b q^{2} ; q^{2}\right)_{k}\left(q^{2} ; q^{2}\right)_{k}} b^{k} \\
& \equiv \frac{(b / q)^{(n-1) / 2}\left(q^{2} / b ; q^{2}\right)_{(n-1) / 2}}{\left(b q^{2} ; q^{2}\right)_{(n-1) / 2}} \frac{\left(b-q^{n}\right)\left(a b-1-a^{2}+a q^{n}\right)}{(a-b)(1-a b)}[n] \\
& \quad+\frac{\left(q ; q^{2}\right)_{(n-1) / 2}^{2}}{\left(a q^{2} ; q^{2}\right)_{(n-1) / 2}\left(q^{2} / a ; q^{2}\right)_{(n-1) / 2}} \frac{\left(1-a q^{n}\right)\left(a-q^{n}\right)}{(a-b)(1-a b)}[n]
\end{aligned}
$$

modulo $[n]\left(1-a q^{n}\right)\left(a-q^{n}\right)\left(b-q^{n}\right)$.

Moreover, by (2.1) and (2.2) we have

$$
\frac{\left(q ; q^{2}\right)_{(n-1) / 2}^{2}}{\left(a q, q^{2}\right)_{(n-1) / 2}\left(q / a, q^{2}\right)_{(n-1) / 2}} \equiv \frac{n(1-a) a^{(n-1) / 2}}{\left(1-a^{n}\right) q^{(n-1) / 2}} \quad\left(\bmod \Phi_{n}(q)\right) .
$$

It is easy to see that the limit of $b-q^{n}$ as $b \rightarrow 1$ has the factor $\Phi_{n}(q)$. Meanwhile, the factor $\left(b q^{2} ; q^{2}\right)_{(n-1) / d}$ in the denominator of the left-hand side of $(2.8)$ as $b \rightarrow 1$ is relatively prime to $\Phi_{n}(q)$. Thus, letting $b \rightarrow 1$ in (2.8) and applying (2.9), we conclude that (1.4) is true modulo $\Phi_{n}(q)^{2}\left(1-a q^{n}\right)\left(a-q^{n}\right)$. Here we used the following relation:

$$
\left(1-q^{n}\right)\left(1+a^{2}-a-a q^{n}\right)=(1-a)^{2}+\left(1-a q^{n}\right)\left(a-q^{n}\right) .
$$

Note that (2.4) is also true for $b=1$. That is, the $q$-congruence (1.4) is true modulo $[n]$. The proof then follows from the fact that the least common multiple of $\Phi_{n}(q)^{2}(1-$ $\left.a q^{n}\right)\left(a-q^{n}\right)$ and $[n]$ is $[n] \Phi_{n}(q)\left(1-a q^{n}\right)\left(a-q^{n}\right)$.

\section{Proof of Theorem 1.2}

Similarly as before, we need the following lemma, which was proved by the author and Zudilin [14, Theorem 4.5].

Lemma 3.1. Let $n \equiv r(\bmod 4)$ be a positive odd integer, where $r= \pm 1$. Then, modulo $[n]\left(1-a q^{n}\right)\left(a-q^{n}\right)$,

$$
\begin{aligned}
& \sum_{k=0}^{(n-1) / 2}[6 k+1] \frac{\left(a q ; q^{2}\right)_{k}\left(q / a ; q^{2}\right)_{k}\left(q ; q^{2}\right)_{k}\left(q^{2} / b ; q^{4}\right)_{k} b^{k} q^{k^{2}}}{\left(a q^{4} ; q^{4}\right)_{k}\left(q^{4} / a ; q^{4}\right)_{k}\left(q^{4} ; q^{4}\right)_{k}\left(b q ; q^{2}\right)_{k}} \\
& \equiv \frac{\left(q^{2+r} / b ; q^{4}\right)_{(n-r) / 4}}{\left(b q^{2+r} ; q^{4}\right)_{(n-r) / 4}} b^{(n-r) / 4}(-q)^{(1-n) / 2}[n] .
\end{aligned}
$$

We find that the left-hand side of (3.1) has a simple formula modulo $b-q^{2 n}$. 
Lemma 3.2. Let $n$ be a positive odd integer. Then, modulo $b-q^{2 n}$,

$$
\sum_{k=0}^{(n-1) / 2}[6 k+1] \frac{\left(a q ; q^{2}\right)_{k}\left(q / a ; q^{2}\right)_{k}\left(q ; q^{2}\right)_{k}\left(q^{2} / b ; q^{4}\right)_{k} b^{k} q^{k^{2}}}{\left(a q^{4} ; q^{4}\right)_{k}\left(q^{4} / a ; q^{4}\right)_{k}\left(q^{4} ; q^{4}\right)_{k}\left(b q ; q^{2}\right)_{k}} \equiv \frac{\left(q ; q^{2}\right)_{(n-1) / 2}\left(q^{n+2} ; q^{2}\right)_{(n-1) / 2}[n]}{\left(a q^{4} ; q^{4}\right)_{(n-1) / 2}\left(q^{4} / a ; q^{4}\right)_{(n-1) / 2}} .
$$

Proof. The derivation of (3.1) in [14] uses the formula [18, Equation (4.6)]:

$$
\begin{aligned}
& \sum_{k=0}^{\infty} \frac{(a ; q)_{k}\left(1-a q^{3 k}\right)(d ; q)_{k}(q / d ; q)_{k}\left(b ; q^{2}\right)_{k}}{\left(q^{2} ; q^{2}\right)_{k}(1-a)\left(a q^{2} / d ; q^{2}\right)_{k}\left(a d q ; q^{2}\right)_{k}(a q / b ; q)_{k}} \frac{a^{k} q^{\left(\begin{array}{c}
k+1 \\
2
\end{array}\right)}}{b^{k}} \\
& \quad=\frac{(a q ; q)_{\infty}\left(a d q / b ; q^{2}\right)_{\infty}\left(a q^{2} / b d ; q^{2}\right)_{\infty}}{(a q / b ; q)_{\infty}\left(a q^{2} / d ; q^{2}\right)_{\infty}\left(a d q ; q^{2}\right)_{\infty}}
\end{aligned}
$$

Letting $q \mapsto q^{2}$ and taking $a=q, d=a q$ and $b \mapsto q^{2} / b$, we are led to

$$
\begin{aligned}
& \sum_{k=0}^{\infty}[6 k+1] \frac{\left(a q ; q^{2}\right)_{k}\left(q / a ; q^{2}\right)_{k}\left(q ; q^{2}\right)_{k}\left(q^{2} / b ; q^{4}\right)_{k} b^{k} q^{k^{2}}}{\left(a q^{4} ; q^{4}\right)_{k}\left(q^{4} / a ; q^{4}\right)_{k}\left(q^{4} ; q^{4}\right)_{k}\left(b q ; q^{2}\right)_{k}} \\
& \quad=\frac{\left(q^{3} ; q^{2}\right)_{\infty}\left(a b q^{2} ; q^{4}\right)_{\infty}\left(b q^{2} / a ; q^{4}\right)_{\infty}}{\left(b q ; q^{2}\right)_{\infty}\left(a q^{4} ; q^{4}\right)_{\infty}\left(q^{4} / a ; q^{4}\right)_{\infty}}
\end{aligned}
$$

Substituting $b=q^{2 n}$ into the above identity, we obtain

$$
\sum_{k=0}^{(n-1) / 2}[6 k+1] \frac{\left(a q ; q^{2}\right)_{k}\left(q / a ; q^{2}\right)_{k}\left(q ; q^{2}\right)_{k}\left(q^{2-2 n} ; q^{4}\right)_{k} q^{k^{2}+n k}}{\left(a q^{4} ; q^{4}\right)_{k}\left(q^{4} / a ; q^{4}\right)_{k}\left(q^{4} ; q^{4}\right)_{k}\left(q^{n+1} ; q^{2}\right)_{k}}=\frac{\left(q^{3} ; q^{2}\right)_{n-1}}{\left(a q^{4} ; q^{4}\right)_{(n-1) / 2}\left(q^{4} / a ; q^{4}\right)_{(n-1) / 2}} .
$$

Namely, the $q$-congruence (3.2) holds.

Proof of Theorem 1.2. Suppose that $n \equiv r(\bmod 4)$ with $r= \pm 1$. By (3.1) and (3.2) with $b \mapsto b^{2}$, we have the following $q$-congruence modulo $[n]\left(1-a q^{n}\right)\left(a-q^{n}\right)\left(b-q^{n}\right)$ :

$$
\begin{aligned}
& \sum_{k=0}^{(n-1) / 2}[6 k+1] \frac{\left(a q ; q^{2}\right)_{k}\left(q / a ; q^{2}\right)_{k}\left(q ; q^{2}\right)_{k}\left(q^{2} / b^{2} ; q^{4}\right)_{k} b^{2 k} q^{k^{2}}}{\left(a q^{4} ; q^{4}\right)_{k}\left(q^{4} / a ; q^{4}\right)_{k}\left(q^{4} ; q^{4}\right)_{k}\left(b^{2} q ; q^{2}\right)_{k}} \\
& \equiv \frac{\left(q^{2+r} / b ; q^{4}\right)_{(n-r) / 4}}{\left(b q^{2+r} ; q^{4}\right)_{(n-r) / 4}} b^{(n-r) / 4}(-q)^{(1-n) / 2} \frac{\left(b-q^{n}\right)\left(a b-1-a^{2}+a q^{n}\right)}{(a-b)(1-a b)}[n] \\
& \quad+\frac{\left(q ; q^{2}\right)_{(n-1) / 2}\left(q^{n+2} ; q^{2}\right)_{(n-1) / 2}}{\left(a q^{4} ; q^{4}\right)_{(n-1) / 2}\left(q^{4} / a ; q^{4}\right)_{(n-1) / 2}} \frac{\left(1-a q^{n}\right)\left(a-q^{n}\right)}{(a-b)(1-a b)}[n]
\end{aligned}
$$

where we have used the $q$-congruences (2.6) $)$ and (2.7). It is clear that $q^{n} \equiv 1\left(\bmod \Phi_{n}(q)\right)$. By (2.1) (with $q \mapsto q^{2}$ ) and (2.3) (with $a=1$ ), we have

$$
\begin{aligned}
\frac{\left(q ; q^{2}\right)_{(n-1) / 2}\left(q^{n+2} ; q^{2}\right)_{(n-1) / 2}}{\left(a q^{4} ; q^{4}\right)_{(n-1) / 2}\left(q^{4} / a ; q^{4}\right)_{(n-1) / 2}} & \equiv \frac{\left(q ; q^{2}\right)_{(n-1) / 2}\left(q^{2} ; q^{2}\right)_{(n-1) / 2}}{\left(a q^{4} ; q^{4}\right)_{(n-1) / 2}\left(q^{4} / a ; q^{4}\right)_{(n-1) / 2}} \\
& \equiv(-q)^{(1-n) / 2} \frac{n(1-a) a^{(n-1) / 2}}{1-a^{n}} \quad\left(\bmod \Phi_{n}(q)\right) .
\end{aligned}
$$


Like the proof of Theorem 1.1, the limit of $b-q^{n}$ as $b \rightarrow 1$ has the factor $\Phi_{n}(q)$ and the factor $\left(b^{2} q ; q^{2}\right)_{(n-1) / 2}$ in the denominator of (3.4) as $b \rightarrow 1$ is coprime with $\Phi_{n}(q)$. Thus, letting $b \rightarrow 1$ in (2.8) and applying (3.5) and (2.10), we conclude that (1.6) is true modulo $\Phi_{n}(q)^{2}\left(1-a q^{n}\right)\left(a-q^{n}\right)$. Further, the $q$-congruence (3.1) also holds for $b=1$, i.e., the $q$-congruence (1.6) is true modulo $[n]$. This completes the proof.

\section{Proof of Theorem 1.3}

We first give the following $q$-congruence, which follows from the $c \rightarrow 0$ case of $[10$, Theorem 6.1] (see also [14, Conjecture 4.6]).

Lemma 4.1. Let $n$ be a positive odd integer. Then, modulo $[n]\left(1-a q^{n}\right)\left(a-q^{n}\right)$,

$$
\sum_{k=0}^{n-1}[3 k+1] \frac{\left(a q ; q^{2}\right)_{k}\left(q / a ; q^{2}\right)_{k}\left(q ; q^{2}\right)_{k}(q / b ; q)_{k} b^{k} q^{-\left(\begin{array}{c}
k+1 \\
2
\end{array}\right)}}{(a q ; q)_{k}(q / a ; q)_{k}(q ; q)_{k}\left(b q^{2} ; q^{2}\right)_{k}} \equiv \frac{(b / q)^{(n-1) / 2}\left(q^{2} / b ; q^{2}\right)_{(n-1) / 2}}{\left(b q^{2} ; q^{2}\right)_{(n-1) / 2}}[n] .
$$

We also have a simple $q$-congruence for the left-hand side of (4.1) modulo $b-q^{n}$.

Lemma 4.2. Let $n$ be a positive odd integer. Then, modulo $b-q^{n}$,

$$
\sum_{k=0}^{n-1}[3 k+1] \frac{\left(a q ; q^{2}\right)_{k}\left(q / a ; q^{2}\right)_{k}\left(q ; q^{2}\right)_{k}(q / b ; q)_{k} b^{k} q^{-\left(\begin{array}{c}
k+1 \\
2
\end{array}\right)}}{(a q ; q)_{k}(q / a ; q)_{k}(q ; q)_{k}\left(b q^{2} ; q^{2}\right)_{k}} \equiv \frac{\left(q ; q^{2}\right)_{(n-1) / 2}^{2}[n]}{\left(a q^{2} ; q^{2}\right)_{(n-1) / 2}\left(q^{2} / a ; q^{2}\right)_{(n-1) / 2}} .
$$

Proof. Using the transformation formula [2, Equation (3.8.13)] and the Pfaff-Saalschütz theorem [2, Appendix (II.12)], in the sketch of proof of [14, Theorem 4.8] the author and Zudilin gave

$$
\begin{aligned}
& \sum_{k=0}^{2 N}[3 k+1] \frac{\left(a q ; q^{2}\right)_{k}\left(q / a ; q^{2}\right)_{k}\left(q ; q^{2}\right)_{k}(q / b ; q)_{k}\left(q^{-2 N} ; q\right)_{k}\left(b q^{1+2 N} ; q\right)_{k} q^{k}}{(a q ; q)_{k}(q / a ; q)_{k}(q ; q)_{k}\left(b q^{2} ; q^{2}\right)_{k}\left(q^{3+2 N} ; q^{2}\right)_{k}\left(q^{2-2 N} / b ; q^{2}\right)_{k}} \\
& \quad=\frac{\left(q^{1-2 N} ; q^{2}\right)_{\infty}\left(q^{2} / b ; q^{2}\right)_{\infty}\left(b q^{2+2 N} ; q^{2}\right)_{\infty}}{(1-q)\left(q^{3+2 N} ; q^{2}\right)_{\infty}\left(q^{2-2 N} / b ; q^{2}\right)_{\infty}\left(b q^{2} ; q^{2}\right)_{\infty}} \frac{\left(a b q ; q^{2}\right)_{N}\left(a q^{1-2 N} / b ; q^{2}\right)_{N}}{\left(a q^{2} ; q^{2}\right)_{N}\left(a q^{-2 N} ; q^{2}\right)_{N}} \\
& \quad=\frac{\left(q ; q^{2}\right)_{N}^{2}\left(a b q ; q^{2}\right)_{N}\left(b q / a ; q^{2}\right)_{N}[2 N+1]}{\left(b ; q^{2}\right)_{N}\left(b q^{2} ; q^{2}\right)_{N}\left(a q^{2} ; q^{2}\right)_{N}\left(q^{2} / a ; q^{2}\right)_{N}}
\end{aligned}
$$

(we correct a typo in the first equality here). Letting $N=(n-1) / 2$ and $b \rightarrow 0$ in the above identity, we are led to

$$
\sum_{k=0}^{n-1}[3 k+1] \frac{\left(a q ; q^{2}\right)_{k}\left(q / a ; q^{2}\right)_{k}\left(q ; q^{2}\right)_{k}\left(q^{1-n} ; q\right)_{k} q^{n k-\left(\begin{array}{c}
k+1 \\
2
\end{array}\right)}}{(a q ; q)_{k}(q / a ; q)_{k}(q ; q)_{k}\left(q^{n+2} ; q^{2}\right)_{k}}=\frac{\left(q ; q^{2}\right)_{(n-1) / 2}^{2}[n]}{\left(a q^{2} ; q^{2}\right)_{(n-1) / 2}\left(q^{2} / a ; q^{2}\right)_{(n-1) / 2}} .
$$

Namely, the desired $q$-congruence holds. 
Proof of Theorem 1.3. From (4.1) and (4.2) we can deduce that, modulo $[n]\left(1-a q^{n}\right)(a-$ $\left.q^{n}\right)\left(b-q^{n}\right)$

$$
\begin{gathered}
\sum_{k=0}^{n-1}[3 k+1] \frac{\left(a q ; q^{2}\right)_{k}\left(q / a ; q^{2}\right)_{k}\left(q ; q^{2}\right)_{k}(q / b ; q)_{k} b^{k} q^{-\left(\begin{array}{c}
k+1 \\
2
\end{array}\right)}}{(a q ; q)_{k}(q / a ; q)_{k}(q ; q)_{k}\left(b q^{2} ; q^{2}\right)_{k}} \\
\equiv \frac{(b / q)^{(n-1) / 2}\left(q^{2} / b ; q^{2}\right)_{(n-1) / 2}}{\left(b q^{2} ; q^{2}\right)_{(n-1) / 2}} \frac{\left(b-q^{n}\right)\left(a b-1-a^{2}+a q^{n}\right)}{(a-b)(1-a b)}[n] \\
+\frac{\left(q ; q^{2}\right)_{(n-1) / 2}^{2}}{\left(a q^{2} ; q^{2}\right)_{(n-1) / 2}\left(q^{2} / a ; q^{2}\right)_{(n-1) / 2}} \frac{\left(1-a q^{n}\right)\left(a-q^{n}\right)}{(a-b)(1-a b)}[n]
\end{gathered}
$$

where we have utilized (2.6) and (2.7). Note that the right-hand sides of (2.8) and (4.3) are exactly the same. Thus, letting $b \rightarrow 1$ in (4.3), we arrive at (1.8).

\section{Concluding remarks and open problems}

We first give the following $q$-congruence related to Theorem 1.1. To the best of our knowledge, this is the first $q$-congruence modulo $[n] \Phi_{n}(q)\left(1-a q^{n}\right)\left(a-q^{n}\right)\left(b-q^{n}\right)$ in the literature.

Theorem 5.1. Let $n$ be a positive odd integer. Then, modulo $[n] \Phi_{n}(q)\left(1-a q^{n}\right)\left(a-q^{n}\right)(b-$ $\left.q^{n}\right)$,

$$
\begin{aligned}
& \sum_{k=0}^{(n-1) / 2}[4 k+1] \frac{\left(a q ; q^{2}\right)_{k}\left(q / a ; q^{2}\right)_{k}\left(q / b ; q^{2}\right)_{k}\left(q ; q^{2}\right)_{k}}{\left(a q^{2} ; q^{2}\right)_{k}\left(q^{2} / a ; q^{2}\right)_{k}\left(b q^{2} ; q^{2}\right)_{k}\left(q^{2} ; q^{2}\right)_{k}} b^{k} \\
& \quad \equiv \sum_{k=0}^{n-1}[4 k+1] \frac{\left(a q ; q^{2}\right)_{k}\left(q / a ; q^{2}\right)_{k}\left(q / b ; q^{2}\right)_{k}\left(q ; q^{2}\right)_{k}}{\left(a q^{2} ; q^{2}\right)_{k}\left(q^{2} / a ; q^{2}\right)_{k}\left(b q^{2} ; q^{2}\right)_{k}\left(q^{2} ; q^{2}\right)_{k}} b^{k}
\end{aligned}
$$

In particular, we have

$$
\sum_{k=0}^{(n-1) / 2}[4 k+1] \frac{\left(q ; q^{2}\right)_{k}^{4}}{\left(q^{2} ; q^{2}\right)_{k}^{4}} \equiv \sum_{k=0}^{n-1}[4 k+1] \frac{\left(q ; q^{2}\right)_{k}^{4}}{\left(q^{2} ; q^{2}\right)_{k}^{4}} \quad\left(\bmod [n] \Phi_{n}(q)^{4}\right)
$$

Proof. By [2, Appendix (I.11)], we have

$$
\frac{(a ; q)_{n-k}}{(b ; q)_{n-k}}=\frac{(a ; q)_{n}\left(q^{1-n} / b ; q\right)_{k}}{(b ; q)_{n}\left(q^{1-n} / a ; q\right)_{k}}\left(\frac{b}{a}\right)^{k} \equiv \frac{(a ; q)_{n}(q / b ; q)_{k}}{(b ; q)_{n}(q / a ; q)_{k}}\left(\frac{b}{a}\right)^{k} \quad\left(\bmod \Phi_{n}(q)\right) .
$$


It follows that

$$
\begin{aligned}
& \sum_{k=(n+1) / 2}^{n-1}[4 k+1] \frac{\left(a q ; q^{2}\right)_{k}\left(q / a ; q^{2}\right)_{k}\left(q / b ; q^{2}\right)_{k}\left(q ; q^{2}\right)_{k}}{\left(a q^{2} ; q^{2}\right)_{k}\left(q^{2} / a ; q^{2}\right)_{k}\left(b q^{2} ; q^{2}\right)_{k}\left(q^{2} ; q^{2}\right)_{k}} b^{k} \\
& =\sum_{k=1}^{(n-1) / 2}[4(n-k)+1] \frac{\left(a q ; q^{2}\right)_{n-k}\left(q / a ; q^{2}\right)_{n-k}\left(q / b ; q^{2}\right)_{n-k}\left(q ; q^{2}\right)_{n-k}}{\left(a q^{2} ; q^{2}\right)_{n-k}\left(q^{2} / a ; q^{2}\right)_{n-k}\left(b q^{2} ; q^{2}\right)_{n-k}\left(q^{2} ; q^{2}\right)_{n-k}} b^{n-k} \\
& \equiv \frac{\left(a q ; q^{2}\right)_{n}\left(q / a ; q^{2}\right)_{n}\left(q / b ; q^{2}\right)_{n}\left(q ; q^{2}\right)_{n} b^{n}}{\left(a q^{2} ; q^{2}\right)_{n}\left(q^{2} / a ; q^{2}\right)_{n}\left(b q^{2} ; q^{2}\right)_{n}\left(q^{2} ; q^{2}\right)_{n-1}} \\
& \quad \times \sum_{k=1}^{(n-1) / 2}[1-4 k] \frac{\left(1 / a ; q^{2}\right)_{k}\left(a ; q^{2}\right)_{k}\left(1 / b ; q^{2}\right)_{k}\left(q^{2} ; q^{2}\right)_{k-1}}{\left(q / a ; q^{2}\right)_{k}\left(a q ; q^{2}\right)_{k}\left(b q ; q^{2}\right)_{k}\left(q ; q^{2}\right)_{k}} b^{4 k} \quad\left(\bmod \Phi_{n}(q)^{2}\right),
\end{aligned}
$$

where we have used the fact $\left(q ; q^{2}\right)_{n} \equiv 0\left(\bmod \Phi_{n}(q)\right)$. Like [10, Lemma 3.1], we can show that

$$
\begin{aligned}
& \frac{\left(a ; q^{2}\right)_{(n+1) / 2-k}}{\left(q / a ; q^{2}\right)_{(n+1) / 2-k}} \equiv(-a)^{(n+1) / 2-2 k} \frac{\left(a ; q^{2}\right)_{k}}{\left(q / a ; q^{2}\right)_{k}} q^{\left(n^{2}-1\right) / 4+k} \quad\left(\bmod \Phi_{n}(q)\right), \\
& \frac{\left(q^{2} ; q^{2}\right)_{(n+1) / 2-k-1}}{\left(q ; q^{2}\right)_{(n+1) / 2-k}} \equiv(-1)^{(n+1) / 2} \frac{\left(q^{2} ; q^{2}\right)_{k-1}}{\left(q ; q^{2}\right)_{k}} q^{\left(n^{2}-1\right) / 4+k}
\end{aligned}
$$

for $1 \leqslant k \leqslant(n-1) / 2$, and so the $k$-th and $(n+1) / 2-k$-th terms in the summation of the right-hand side of (5.3) cancel each other modulo $\Phi_{n}(q)$. Noticing that the fraction before the summation is congruent to 0 modulo $\Phi_{n}(q)$ too, we conclude that the right-hand side of (5.3) is congruent to 0 modulo $\Phi_{n}(q)^{2}$. This proves (5.1) modulo $\Phi_{n}(q)^{2}$. By the proof of Theorem 1.1, we know that (5.1) also holds modulo $[n]\left(1-a q^{n}\right)\left(a-q^{n}\right)\left(b-q^{n}\right)$. This completes the proof.

Letting $n=p^{r}$ be an odd prime power and letting $q \rightarrow 1$ in (5.2), we obtain

$$
\sum_{k=0}^{\left(p^{r}-1\right) / 2}(4 k+1) \frac{\left(\frac{1}{2}\right)_{k}^{4}}{k !^{4}} \equiv \sum_{k=0}^{p^{r}-1}(4 k+1) \frac{\left(\frac{1}{2}\right)_{k}^{4}}{k !^{4}} \quad\left(\bmod p^{r+4}\right) .
$$

Recall that the Bernoulli numbers $B_{n}$ are defined as follows:

$$
B_{0}=1, \quad \sum_{k=0}^{n}\left(\begin{array}{c}
n+1 \\
k
\end{array}\right) B_{k}=0, \quad \text { for } n=1,2, \ldots
$$

Based on numerical calculations, we would like to propose the following conjecture.

Conjecture 5.2. Let $p>3$ be a prime and $r$ a positive integer. Then

$$
\sum_{k=0}^{\left(p^{r}-1\right) / 2}(4 k+1) \frac{\left(\frac{1}{2}\right)_{k}^{4}}{k !^{4}} \equiv p^{r}+\frac{7}{6} B_{p-3} p^{r+3} \quad\left(\bmod p^{r+4}\right) .
$$


Note that Sun [20, Conjecture 5.1(ii)] conjectured that, for any prime $p>3$ and integer $r \geqslant 1$,

$$
\sum_{k=0}^{p^{r}-1}(3 k+1) \frac{\left(\frac{1}{2}\right)_{k}^{3}}{k !^{3}} 2^{2 k} \equiv p^{r}+\frac{7}{6} B_{p-3} p^{r+3} \quad\left(\bmod p^{r+4}\right) .
$$

Since the right-hand sides of $(\underline{5.5})$ and (5.6) are exactly the same, it is natural to raise the following new conjecture (which is also valid for $p=3$ ).

Conjecture 5.3. Let $p$ be an odd prime and $r$ a positive integer. Then

$$
\sum_{k=0}^{\left(p^{r}-1\right) / 2}(4 k+1) \frac{\left(\frac{1}{2}\right)_{k}^{4}}{k !^{4}} \equiv \sum_{k=0}^{p^{r}-1}(3 k+1) \frac{\left(\frac{1}{2}\right)_{k}^{3}}{k !^{3}} 2^{2 k} \quad\left(\bmod p^{r+4}\right)
$$

Although it is very difficult to give $q$-analogues of supercongruences involving Bernoulli numbers, we find a $q$-analogue of (5.7), which is included in the following conjecture.

Conjecture 5.4. Let $n$ be a positive odd integer. Then, modulo $[n] \Phi_{n}(q)\left(1-a q^{n}\right)(a-$ $\left.q^{n}\right)\left(b-q^{n}\right)$,

$$
\begin{aligned}
& \sum_{k=0}^{(n-1) / 2}[4 k+1] \frac{\left(a q ; q^{2}\right)_{k}\left(q / a ; q^{2}\right)_{k}\left(q / b ; q^{2}\right)_{k}\left(q ; q^{2}\right)_{k}}{\left(a q^{2} ; q^{2}\right)_{k}\left(q^{2} / a ; q^{2}\right)_{k}\left(b q^{2} ; q^{2}\right)_{k}\left(q^{2} ; q^{2}\right)_{k}} b^{k} \\
& \quad \equiv \sum_{k=0}^{n-1}[3 k+1] \frac{\left(a q ; q^{2}\right)_{k}\left(q / a ; q^{2}\right)_{k}\left(q ; q^{2}\right)_{k}(q / b ; q)_{k} b^{k} q^{-\left(\begin{array}{c}
k+1 \\
2
\end{array}\right)}}{(a q ; q)_{k}(q / a ; q)_{k}(q ; q)_{k}\left(b q^{2} ; q^{2}\right)_{k}}
\end{aligned}
$$

In particular, we have

$$
\sum_{k=0}^{(n-1) / 2}[4 k+1] \frac{\left(q ; q^{2}\right)_{k}^{4}}{\left(q^{2} ; q^{2}\right)_{k}^{4}} \equiv \sum_{k=0}^{n-1}[3 k+1] \frac{\left(q ; q^{2}\right)_{k}^{3} q^{-\left(\begin{array}{c}
k+1 \\
2
\end{array}\right)}}{(q ; q)_{k}^{2}\left(q^{2} ; q^{2}\right)_{k}} \quad\left(\bmod [n] \Phi_{n}(q)^{4}\right)
$$

It is easy to see that (5.7) follows from (5.9) by taking $n=p^{r}$ and $q \rightarrow 1$. Note that the $q$-congruence (5.8) is true modulo $[n]\left(1-a q^{n}\right)\left(a-q^{n}\right)\left(b-q^{n}\right)$ by (2.8) and (4.3). Thus, to prove Conjecture 5.4 it suffices to show that (5.8) is true modulo $\Phi_{n}(q)^{2}$.

Acknowledgment. This work was partially supported by the National Natural Science Foundation of China (grant 11771175).

\section{References}

[1] B. Berndt, Ramanujan's Notebooks, Part IV, Springer-Verlag, New York, 1994.

[2] G. Gasper and M. Rahman, Basic hypergeometric series, second edition, Encyclopedia of Mathematics and Its Applications 96, Cambridge University Press, Cambridge, 2004. 
[3] O. Gorodetsky, $q$-Congruences, with applications to supercongruences and the cyclic sieving phenomenon, Int. J. Number Theory 15 (2019), 1919-1968.

[4] V.J.W. Guo, A q-analogue of a Ramanujan-type supercongruence involving central binomial coefficients, J. Math. Anal. Appl. 458 (2018), 590-600.

[5] V.J.W. Guo, A q-analogue of the (J.2) supercongruence of Van Hamme, J. Math. Anal. Appl. 466 (2018), 776-788.

[6] V.J.W. Guo, Common q-analogues of some different supercongruences, Results Math. 74 (2019), Art. 131.

[7] V.J.W. Guo, q-Analogues of two "divergent" Ramanujan-type supercongruences, Ramanujan J., in press; https://doi.org/10.1007/s11139-019-00161-0

[8] V.J.W. Guo and J.-C. Liu, q-Analogues of two Ramanujan-type formulas for $1 / \pi$, J. Difference Equ. Appl. 24 (2018), 1368-1373.

[9] V. J. W. Guo, H. Pan and Y. Zhang, The Rodriguez-Villegas type congruences for truncated $q$-hypergeometric functions, J. Number Theory 174 (2017), 358-368.

[10] V.J.W. Guo and M.J. Schlosser, Some $q$-supercongruences from transformation formulas for basic hypergeometric series, Constr. Approx., to appear.

[11] V.J.W. Guo and M.J. Schlosser, Some new $q$-congruences for truncated basic hypergeometric series: even powers, Results Math. 75 (2020), Art. 1.

[12] V.J.W. Guo and M.J. Schlosser, A family of $q$-hypergeometric congruences modulo the fourth power of a cyclotomic polynomial, Isarel J. Math., to appear.

[13] V.J.W. Guo and S.-D. Wang, Some congruences involving fourth powers of central q-binomial coefficients, Proc. Roy. Soc. Edinburgh Sect. A, https://doi.org/10.1017/prm.2018.96

[14] V.J.W. Guo and W. Zudilin, A q-microscope for supercongruences, Adv. Math. 346 (2019), 329-358.

[15] L. Long, Hypergeometric evaluation identities and supercongruences, Pacific J. Math. 249 (2011), 405-418.

[16] H.-X. Ni and H. Pan, Some symmetric q-congruences modulo the square of a cyclotomic polynomial, J. Math. Anal. Appl. 481 (2020), Art. 123372.

[17] R. Osburn and W. Zudilin, On the (K.2) supercongruence of Van Hamme, J. Math. Anal. Appl. 433 (2016), 706-711.

[18] M. Rahman, Some quadratic and cubic summation formulas for basic hypergeometric series, Canad. J. Math. 45 (1993), 394-411.

[19] S. Ramanujan, Modular equations and approximations to $\pi$, Quart. J. Math. Oxford Ser. (2) 45 (1914), 350-372.

[20] Z.-W. Sun, Super congruences and Euler numbers, Sci. China Math. 54 (2011), 2509-2535.

[21] A. Straub, Supercongruences for polynomial analogs of the Apéry numbers, Proc. Amer. Math. Soc. 147 (2019), 1023-1036.

[22] H. Swisher, On the supercongruence conjectures of van Hamme, Res. Math. Sci. 2 (2015), Art. 18.

[23] R. Tauraso, Some $q$-analogs of congruences for central binomial sums, Colloq. Math. 133 (2013), 133-143. 
[24] L. Van Hamme, Some conjectures concerning partial sums of generalized hypergeometric series, in: p-Adic functional analysis (Nijmegen, 1996), Lecture Notes in Pure and Appl. Math. 192, Dekker, New York, 1997, pp. 223-236.

[25] H.S. Wilf and D. Zeilberger, An algorithmic proof theory for hypergeometric (ordinary and "q") multisum/integral identities, Invent. Math. 108 (1992), 575-633.

[26] W. Zudilin, Ramanujan-type supercongruences, J. Number Theory 129 (2009), 1848-1857. 